\section{Intracranial bleeding under dual}

\section{antiplatelet therapy after emergency}

recanalization of intracranial and

extracranial large artery occlusion
Yael Yannick Zdunczyk', Oliver Beuing', Stephan Hause', Jens Neumann', Andrea Breja',

Stefanie Schreiber ${ }^{1}$, H.-Jochen Heinze ${ }^{1,3}$, Anja Lenz ${ }^{2}$, Martin Skalej2, Michael Goertler ${ }^{1,3}$

Vascular and Stroke Center University of Magdeburg

'Department of Neurology

${ }^{2}$ Department of Neuroradiology ${ }^{3}$ Leibniz Institute for Neurobiology

\section{Background}

To assess bleeding risk of (stent-protected) angioplasty (PTA) of extracranial internal carotid (ICA) occlusion with subsequent dual antiplatelet agents in addition to intracranial endovascular thrombectomy in acute anterior circulation stroke.

\section{Methods}

Between 2009 and 2017, 515 consecutive patients admitted to our university stroke center with an acute severe anterior circulation stroke underwent endovascular thrombectomy for intracranial large artery occlusion. Stroke severity was assessed by the NIHSS score at admission. Admission CT was rated according to ASPECTS and controlled after 24 hours, 4 days, and in case of patients' clinical worsening. Functional outcome was evaluated after 3 months by the modified Rankin scale (unfavorable outcome, 3 to 6).

\section{Results}

70 of 515 patients (13.6\%) underwent additional PTA for extracranial ICA occlusion/pseudo-occlusion. PTA was stent-protected in 37 patients because of ulcerative or thrombogenic ICA plaque or insufficient dilatation for thrombectomy-catheter passage by sole PTA. 39 patients received early (within 24 hours) dual antiplatelet agents, i.e. ticagrelor in 28 and clopidogrel in 11 in addition to ASA. In nine patients, clopidogrel was administered as add on to ASA at day 2 to day 7. Patients' characteristics and interventional data are presented in table 1 . Six patients suffered spontaneous symptomatic intracerebral bleeding (table 2). Chi-square test revealed an association of early dual antiplatelet prevention after (stent-protected) PTA and spontaneous symptomatic intracerebral bleeding $(P=0.03)$. However, as all 6 bleedings occurred in the 28 patients with early ticagrelor in addition to ASA $(P=0.003)$ and none in the 11 patients with early clopidogrel (figure), our data may not suggest an increased risk under the combination of ASA and clopidogrel. Bleeding was not associated with an unfavorable outcome, seen in 52 patients (74.3\%). No bleeding association was found for intravenous and intraarterial thrombolysis and ASPECTS at admission.

\section{Conclusions}

Ticagrelor in addition to ASA within 24 hours of intracranial thrombectomy and extracranial (stentprotected) PTA in acute stroke from extra-intracranial tandem occlusion may be associated with an increased risk of spontaneous symptomatic intracerebral bleeding.

\begin{tabular}{|lr|}
\hline Tab. $1 \quad$ Patients' characteristics and endovascular intervention \\
\hline Age, years (mean, SD) & $65.1(11.3)$ \\
\hline Men & $48(68.6 \%)$ \\
\hline Atrial fibrillation & $11(15.7 \%)$ \\
\hline NIHSS-Score at admission (median, IQR) & $19(12-23)$ \\
\hline ASPECTS (median, IQR) & $8.5(8-10)$ \\
\hline Intravenous thrombolysis & $38(54.3 \%)$ \\
\hline General anesthesia & $43(61.4 \%)$ \\
\hline Event to groin-puncture time, hours (mean, SD) & $5.09(3.3)$ \\
\hline Extracranial stent-PTA & $37(52.9 \%)$ \\
\hline Local arterial thrombolysis & $20(28.6 \%)$ \\
\hline Intracranial aspiration (without stent retriever) & $41(58.6 \%)$ \\
\hline Early dual antiplatelets (ASA \& ticagrelor/clopidogrel) & $39(55.7 \%)$ \\
\hline
\end{tabular}

\begin{tabular}{|lr|}
\hline Tab. 2 Outcome variables & \\
\hline Spontaneous symptomatic intracerebral bleeding & $6(8.6 \%)$ \\
\hline Modified Rankin score after 90 days unfavorable $(\geq 3)$ & $52(74.3 \%)$ \\
\hline Mortality after 90 days & $8(11.4 \%)$ \\
\hline
\end{tabular}

Figure Early dual antiplalet therapy and symptomatic intracerebral bleeding

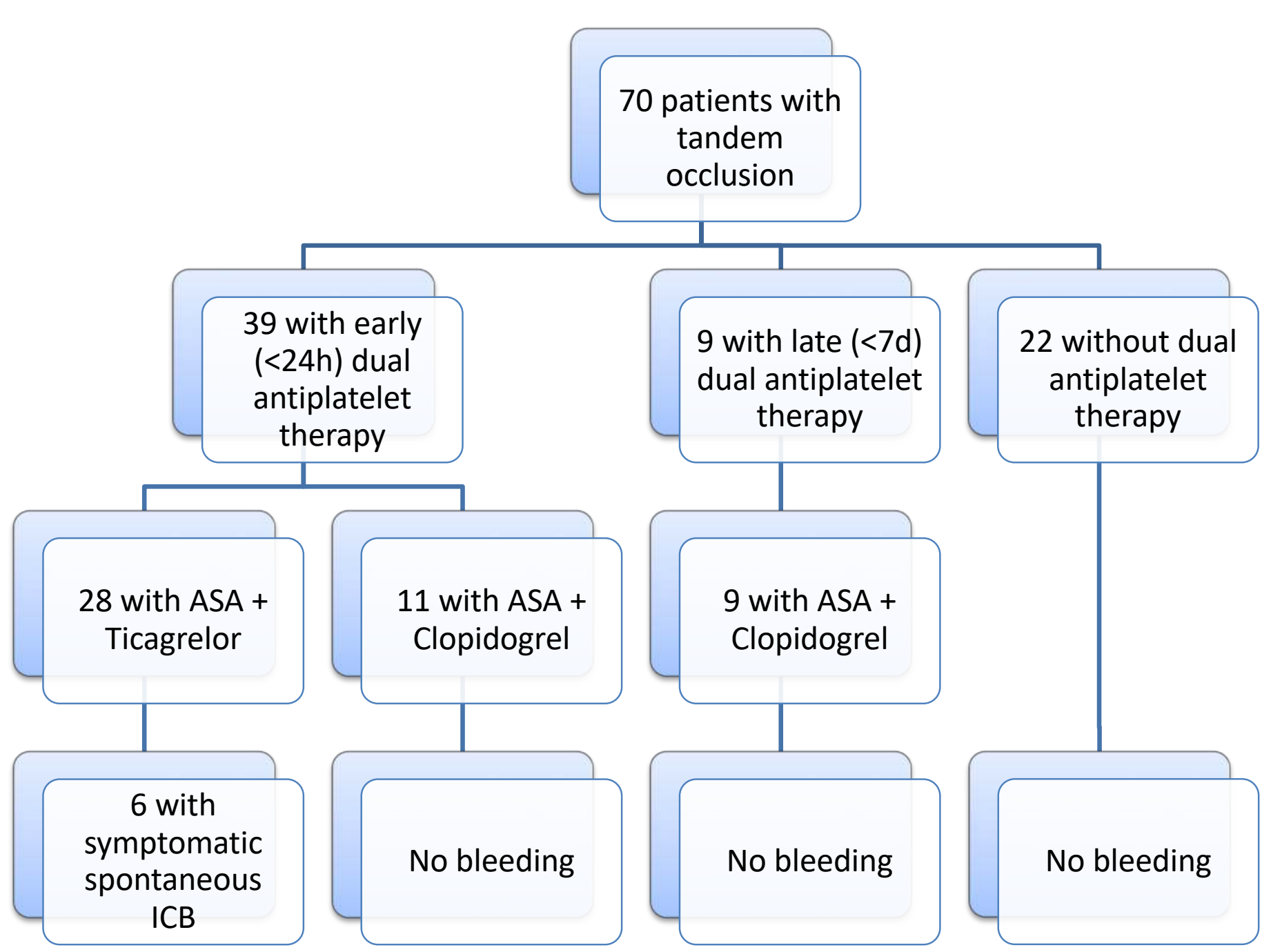

